\title{
Genetic and Environmental Etiology of Infant Hemodynamic Response to Speech Stimuli: A Near-Infrared Spectroscopy Study of Twins
}

\author{
Kunitake Suzuki ${ }^{1}$, Juko Ando ${ }^{2}$ \\ ${ }^{1}$ Faculty of Human Sciences, Osaka University of Human Sciences, Osaka, Japan \\ ${ }^{2}$ Faculty of Letters, Keio University, Tokyo, Japan \\ Email: k-suzuki@kun.ohs.ac.jp, suzuki.res18@gmail.com
}

Received April 14 $4^{\text {th }}, 2013$; revised May 16 $6^{\text {th }}$ 2013; accepted June $13^{\text {th }}, 2013$

\begin{abstract}
Copyright (C) 2013 Kunitake Suzuki, Juko Ando. This is an open access article distributed under the Creative Commons Attribution License, which permits unrestricted use, distribution, and reproduction in any medium, provided the original work is properly cited.
\end{abstract}

\begin{abstract}
For adults and children, genetic and environmental factors are known to affect brain structure and neural activity necessary for conducting various cognitive tasks. However, little is known regarding genetic and environmental contributions to individual differences in neural activity during the first two years of life. Concentrations of oxygenated and deoxygenated hemoglobin were measured bilaterally over temporal areas of 7 monozygotic and 17 dizygotic twin pairs using near-infrared spectroscopy. Results showed that environmental influences on the concentration of hemoglobin were larger than for genetic influences. Significant genetic and environmental influences were detected in different temporal areas. We discuss the genetic and environmental influences on the hemodynamic response to speech stimuli during the first two years of life.
\end{abstract}

Keywords: Environmental Factors; Genetic Factors; Infants; Near-Infrared Spectroscopy; Speech Stimuli; Twins

\section{Introduction}

Cerebral dominance for speech has been a popular research topic, with left cerebral dominance observed within the majority of speech-processing studies (Jansen et al., 2007; Knecht et al., 2000). However, various types of cerebral dominance for speech among infants have been observed. Left (e.g., Bortfeld, Fava, \& Boss, 2009; Kotilahti et al., 2010), right (e.g., DehaeneLambertz et al., 2006; Homae, Watanabe, Nakano, \& Taga, 2007), and no cerebral dominance (Novak, Kurtzberg, Kreuzer, \& Vaughan Jr., 1989) have all been reported. Although these inconsistencies may result from the varied characteristics of speech stimuli (Minagawa-Kawai, Cristià, \& Dupoux, 2011), discrepant cerebral dominance reported in previous studies suggest larger individual differences among infant neural responses to speech as compared to adults. For example, individual differences during the first two years of life are related to the speed of bilateral maturation within temporal brain regions (Chiron et al., 1997), adaptation of neural systems to environmental speech sounds (Cheour et al., 1998), and second language experiences (Conboy \& Kuhl, 2011). Specific factors associated with individual differences in neural responses to speech may have contributed to the varying results observed when testing for cerebral dominance among infants. By accounting for these differences, variance in neural responses to speech within the two hemispheres can be validly determined. In the current study, we decomposed individual differences into genetic and environmental factors as a way to provide new evidence regarding bilateral temporal responses to speech among infants.
Results related to the genetic and environmental influences on neural responses to speech have not been assessed among infants. However, these influences can be indirectly inferred from known genetic and environmental influences on brain structure and language ability. While brain structure is affected by genetic factors more so than environmental factors in both children and adults (e.g., Gilmore et al., 2010; Lenroot et al., 2009), children's language ability is thought to be largely associated with environmental rather than genetic variations (e.g., Alarcón, Plomin, Fulker, Corley, \& DeFries, 1998; Van Hulle, Goldsmith, \& Lemery, 2004). Nevertheless, these studies have suggested that both genetic and environmental factors may influence individual differences in infant neural responses to speech.

We adopted a design using data from monozygotic (MZ) and dizygotic (DZ) twins (twin design; Neale \& Maes, 2004). Estimating the degree to which individual differences are influenced by additive genetic factors, shared environmental factors, and unique environmental factors are possible with this type of design. Additive genetic, shared, and unique environmental factors independently contribute to twin phenotypes. The twin design follows the basic logic that while MZ twins share all their genes, and DZ twins, on average, only share half their genes, the degree to which twins share environments (e.g. family environment) is the same regardless of zygosity. If individual differences in the phenotype are based on additive genetic factors, we would expect that the phenotype similarity between $\mathrm{MZ}$ twin pairs to be twice as large as between DZ twins. Alter- 
natively, if shared environmental factors are more critical, we would expect $\mathrm{MZ}$ and $\mathrm{DZ}$ twin pairs to be equally similar. If unique environmental factors are acting to make co-twins less alike, we would expect phenotypes of MZ pairs to be dissimilar. Measurement errors that randomly affect phenotypes of each twin can cause false dissimilarity between twins, leading to erroneous conclusions regarding unique environmental factors.

Here, we used near-infrared spectroscopy (NIRS; Maki et al., 1995) to acquire hemodynamic responses (oxygenated hemoglobin [oxy-Hb] and deoxygenated hemoglobin [deoxy-Hb]) to speech stimuli within bilateral temporal brain regions because this method is convenient and reliable. In the NIRS technique, continuous near-infrared lasers are used, so that the technique is non-invasive and requires relatively few physical constraints. Moreover, the spatial resolution of the imaging data is relatively high compared to that of an electroencephalogram. The NIRS technique is particularly appropriate for infant research.

In this study, we estimated the degree that genetic and environmental factors influence the concentration of not only oxy$\mathrm{Hb}$ but also deoxy-Hb within each bilateral temporal region. Although the concentration of deoxy-Hb has not always been analyzed in previous studies, changes in the concentration of deoxy- $\mathrm{Hb}$ as opposed to oxy-Hb may indicate neural activation required for conduction tasks (Kato, 2004). Furthermore, how hemoglobin concentrations change among infants is still relatively unknown. In general, decreases in deoxy-Hb concentration are often followed by increases in oxy-Hb. However, other patterns of deoxy-/oxy-Hb concentrations have been reported (Sato et al., 2007); thus, infant oxy- and deoxy-Hb concentrations may contribute to different aspects of neural activation required for a given task.

\section{Methods}

\section{Participants}

Subjects were twin infants recruited via the Tokyo Twin Cohort Project (Ando et al., 2006). All twins were raised together by their biological parents, within their family home, in the Tokyo area. Infants who demonstrated high levels of head motion and crying during the experiment were removed from the study (see data analysis section). To compare results according to zygosity, we discarded any data that were not obtained from both twin infants. With these parameters, data from 48 of 134 twins were available for this study. The zygosity of the remaining 24 twin pairs was assessed via a standard questionnaire (Ooki \& Asaka, 2004). This questionnaire revealed that the sample included $7 \mathrm{MZ}$ twin pairs (4 male-male and 3 female-female pairs) and $17 \mathrm{DZ}$ twin pairs (5 male-male, 5 female-female, and 7 opposite sex pairs). The mean ages of the $\mathrm{MZ}$, same-sex DZ, and opposite-sex DZ twin infants were 12.16 months $(S D=5.06$ months; range $=6.13-17.93$ months $)$, 11.43 months $(S D=4.29$ months; range $=6.00-16.97$ months $)$, and 11.9 months $(S D=4.89$ months; range $=5.77-17.83$ months), respectively. The mean gestational ages of the MZ, same-sex DZ, and opposite-sex DZ twin infants were 35.71 weeks $(S D=1.32$ weeks; range $=33$ - 37 weeks $), 36.20$ weeks $(S D=.89$ weeks; range $=34-37$ weeks $)$, and 34.57 weeks $(S D$ $=3.36$ weeks; range $=29-37$ weeks), respectively. The parents of all infants voluntarily provided informed consent before the infants participated in the study. The ethics committee at the Faculty of Letters, Keio University, approved this study.

\section{Apparatus and Stimuli}

The speech stimuli were recorded by a Japanese woman native to the Tokyo area in order to control for regional speech intonation. The stimuli included news scripts describing the Japanese public pension system and were considered too difficult for infants to understand. Thus, these stimuli could only be processed by brain areas required for non-semantic phonological characteristics of language. The speech stimuli were stored in a digital portable recorder (PMD660, Marantz, Mahwah, N.J., USA) with a condenser microphone for input (KSM27, Shure, Niles, IL, USA) and digitized at a sampling rate of $44.1 \mathrm{~Hz}$ with a 16-bit resolution. The stimuli were edited into 20 -s segments, and noise was reduced using Sound Forge 8 software (Sony, Tokyo, Japan). These stimuli were presented through a sound system that consisted of an amplifier (A100a, Yamaha, Shizuoka, Japan) and a loudspeaker (Reveal, Tannoy, Scotland, UK) at a mean intensity sound pressure level of $68 \mathrm{~dB}$.

\section{Procedures}

Each twin infant was supported by a parent sitting $170 \mathrm{~cm}$ in front of a loudspeaker in a soundproof room. The infants were awake or moderately sleepy without sedation. We presented attractive materials, such as toy figures and cars, between the infant and loudspeaker to maintain infant orientation toward the speaker. The infant was then exposed to speech stimuli during 11 blocks, with each stimulus being presented for $20 \mathrm{~s}$. Stimuli were played backwards during the first block and alternated between forward and backward playback thereafter, resulting in 6 backward (rest) and 5 forward (experimental) blocks. There were no delays between blocks, and the total stimulus exposure time was $220 \mathrm{~s}$. If the infant was composed, this experimental procedure was repeated a second time. If the infant tried to move away from the parent's lap during the speech stimulus exposure, experimental staff attempted to calm the infant. Stimulus presentation was terminated if the infant made large motions or cried but was continued if the infant returned to a calmer state. The co-twin rested outside the soundproof room during the experiment and was unable to hear the speech stimuli.

\section{Near-Infrared Spectroscopy Recordings}

The concentration of oxy- and deoxy-Hb was measured within both temporal brain areas at a $10 \mathrm{~Hz}$ sampling rate with an optical topography system (ETG-7000, Hitachi Medical, Tokyo, Japan). Four probes (two emitting probes and two detecting probes) were attached within each bilateral temporal area in a square alignment. The probe at the lowest and most posterior position within each bilateral temporal area was attached at each $\mathrm{T} 7$ and $\mathrm{T} 8$ position of the international 10-5 system (i.e., the extension of the 10-20 system; Oostenveld \& Praamstra, 2001). Another probe was attached on a line connecting points $\mathrm{T} 7$ and $\mathrm{T} 8$ via the $\mathrm{Cz}$ position. Each probe was separated by a distance of $3 \mathrm{~cm}$, so that penetration depth of near-infrared light into the brain tissue was approximately 3 $\mathrm{cm}$.

\section{Data Analysis}

The concentrations of oxy- and deoxy-Hb were smoothed using a 5-s moving average. If the data for a block included 
artifacts, all data for the block were discarded by visual inspection of oxy- and deoxy-Hb concentration. If the concentration of oxy- and deoxy-Hb at a channel included artifacts during several blocks, then data at that channel were discarded. The remaining concentrations of oxy- and deoxy-Hb were then averaged more than twice, synchronously, to the forward-stimulus blocks at each time point. Infants who had more than two contaminated channels within each bilateral area, and no clean forward-stimulus data, were excluded from any further analyses. According to these criteria, the mean number of forwardstimulus blocks available for analysis was 4.42 . The mean number of channels was 3.90 and 3.75 from the left and right temporal areas, respectively. Among eight cases, data were obtained from two or three channels from each left and right temporal area. All remaining infant pairs provided data from all four channels within the left and right temporal areas.

Mean oxy- and deoxy-Hb waveforms were computed by averaging concentrations of oxy- and deoxy- $\mathrm{Hb}$ at each time point against the last $10 \mathrm{~s}$ of the backward-stimulus block, which served as a baseline period at each channel for each infant. We computed the mean values of oxy- and deoxy- $\mathrm{Hb}$ concentration from 5 to $15 \mathrm{~s}$ after the onset of a forward-stimulus block at each channel for each infant. This period was chosen because we expected to acquire clean concentrations representing the hemodynamic response. The oxy- and deoxy-Hb waveform concentrations during the forward-stimulus period indicate the hemodynamic responses that were relatively affected by neural activation for speech stimuli. Next, the mean values acquired from left-side channels were averaged to acquire representative values of oxy- and deoxy-Hb for the left temporal areas, and the same was repeated for the right-side channels. These representative $\mathrm{Hb}$ values resulted in a signal-to-noise ratio that was greater than the mean oxy- and deoxy-Hb values from each individual channel.

We used a one-sample t-test (two-tailed) to examine whether the hemodynamic response from the temporal areas was significantly different from baseline with a corrected significance level (.05, Bonferroni correction). To test cerebral dominance for speech stimuli, we used a paired t-test to compare the concentrations of hemoglobin between left and right temporal areas. In addition, developmental changes in the concentration of oxyand deoxy-Hb for speech stimuli were tested by regression analysis. Infant age was used as the independent variable, while concentration of hemoglobin was the dependent variable. In the paired t-test and regression analyses, a significance level of .05 was set. Twin and co-twin data were treated as single-participants.

Similarity in the hemoglobin concentration between twin and twin siblings were estimated by calculating Pearson productmoment correlation coefficients for each temporal area. The correlation coefficients were calculated using a double entry method in which each member of the twin pairs was used as both the twin and twin sibling. The degree of genetic and environmental influence on the hemodynamic response was estimated through structural equation modeling (Neale \& Maes, 2004) by OpenMx (version 1.1; Boker et al., 2011). The additive genetic, shared environmental, and unique environmental factors were estimated for each oxy- and deoxy-Hb within each temporal area. Path coefficients from the genetic and environmental factors to each oxy- and deoxy-Hb within left and right temporal areas were estimated. The proportions of genetic and environmental variance to total variance for each hemoglobin type were also estimated.

\section{Results}

Grand average waveforms of the hemodynamic response to speech stimuli across all left and right temporal positions are presented in Figure 1(A). The mean concentrations of oxy- and deoxy-Hb are presented in Figure 1(B). The mean representative oxy- and deoxy-Hb values at bilateral temporal areas did not significantly increase or decrease from the baseline period (left oxy-Hb, $M=.008, S E=.009, t(47)=.94, p=.35$; left deoxy-Hb, $M=.0004, S E=.004, t(47)=.09, p=.92$; right oxy-Hb, $M=.017, S E=.008, t(47)=1.91, p=.06$; right deoxy-Hb, $M=-.008, S E=.007, t(47)=-1.14, p=.26)$. Representative oxy- and deoxy-Hb values across all infants ranged from -.11 to $.21 \mathrm{mMmm}$ and -.07 to $.09 \mathrm{mMmm}$, respectively, in the left temporal area, and from -.13 to $.13 \mathrm{mMmm}$ and -.13 to $.12 \mathrm{mMmm}$, respectively, in the right temporal area. Paired $\mathrm{t}$-tests showed no differences in the concentrations of hemoglobin between the left and right temporal areas (oxy-Hb, $t(47)$ $=.82, p=.42$; deoxy-Hb, $t(47)=-.94, p=.35)$. No developmental changes in oxy- and deoxy-Hb concentrations within both temporal areas were observed by regression analysis (left oxy-Hb, $F(1,46)=.49, p=.48$; left deoxy-Hb, $F(1,46)=.02, p$ $=.87$; right oxy-Hb, $F(1,46)=.46, p=.49$; right deoxy-Hb, $F(1,46)=.01, p=.90)$.

Pearson's product-moment correlation coefficients for oxy$\mathrm{Hb}$ concentrations from the left and right temporal areas within $\mathrm{MZ}$ pairs were -.24 and .05 , respectively; those for deoxy- $\mathrm{Hb}$ concentrations were .69 and .24 , respectively. Correlation coefficients for oxy-Hb concentrations from the left and right temporal areas for DZ twin pairs were similar, at .19 and .24, respectively; those for deoxy-Hb were .10 and -.29 , respectively. Table 1 shows that large path coefficients from the unique

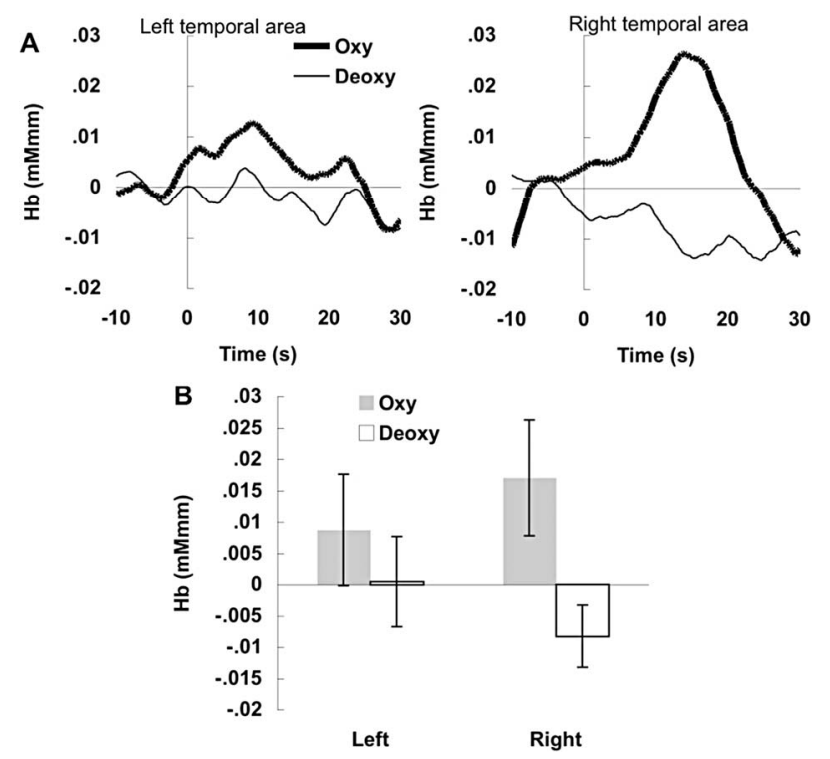

Figure 1.

(A) Grand mean waveform of oxy- and deoxy- $\mathrm{Hb}$ from left and right temporal areas; (B) Mean oxy- and deoxy-Hb concentrations. Note: (A) Waveforms of oxy- and deoxy-Hb from left (A, left) and right (A, right) temporal areas. The horizontal axis displays time in seconds, aligned to the onset of the forward-playing speech stimulus; (B) Error bars indicate standard errors of the mean for oxy- and deoxy-Hb. 
Table 1.

Path coefficients and proportion of variance for each additive genetic, shared environmental, and unique environmental factors.

\begin{tabular}{|c|c|c|c|c|c|c|}
\hline & \multicolumn{3}{|c|}{ Path Coefficient } & \multicolumn{3}{|c|}{ Proportion of Variance } \\
\hline & $\mathrm{a}$ & $\mathrm{c}$ & $\mathrm{e}$ & $\mathrm{a}^{2}$ & $\mathrm{c}^{2}$ & $\mathrm{e}^{2}$ \\
\hline Right oxy-Hb & $\begin{array}{c}-1.18 \mathrm{E}-07 \\
(.0768)\end{array}$ & $\begin{array}{l}.0271^{\dagger} \\
(.0143)\end{array}$ & $\begin{array}{l}.0546^{*} \\
(.0079)\end{array}$ & $3.72 \mathrm{E}-12$ & .20 & .80 \\
\hline Right deoxy-Hb & $\begin{array}{c}-1.53 \mathrm{E}-07 \\
(.0221)\end{array}$ & $\begin{array}{c}-1.78 \mathrm{E}-08 \\
(.0147)\end{array}$ & $\begin{array}{l}.0494^{*} \\
(.0050)\end{array}$ & $9.57 \mathrm{E}-12$ & $1.30 \mathrm{E}-13$ & 1.00 \\
\hline Left oxy-Hb & $\begin{array}{c}-5.27 \mathrm{E}-08 \\
(.0452)\end{array}$ & $\begin{array}{c}.0171 \\
(.0240)\end{array}$ & $\begin{array}{l}.0609^{*} \\
(.0088)\end{array}$ & $6.94 \mathrm{E}-13$ & .07 & .93 \\
\hline Left deoxy-Hb & $\begin{array}{l}.0261^{*} \\
(.0069)\end{array}$ & $\begin{array}{c}5.30 \mathrm{E}-10 \\
(.0178)\end{array}$ & $\begin{array}{l}.0221^{*} \\
(.0057)\end{array}$ & .58 & $2.40 \mathrm{E}-16$ & .42 \\
\hline
\end{tabular}

Note: ${ }^{*} p<.05 ;{ }^{\dagger} p<.1$, Numbers in parentheses indicate standard errors. $\mathrm{a}=$ Additive genetic factor; $\mathrm{c}=$ Shared environmental factor; $\mathrm{e}=$ Unique environmental factor; $\mathrm{a}^{2}$ $=$ Additive genetic variance; $\mathrm{c}^{2}=$ Shared environmental variance; $\mathrm{e}^{2}=$ Unique environmental variance.

environmental factors to each hemoglobin concentration were observed within both bilateral temporal areas.

Shared path coefficients to the oxy-Hb were moderate values within each bilateral temporal area, but statistically significant path coefficients were observed solely for the shared environment to right oxy-Hb. The proportion of shared environmental variance to total variance in oxy-Hb concentration was .07 and .20 within the left and right temporal areas, respectively. In contrast, a significant additive genetic path coefficient was observed solely for left deoxy-Hb concentration. Additive genetic variance largely contributed to the variance in concentration of deoxy-Hb within the left temporal area.

\section{Discussion}

We demonstrated both genetic and environmental influences on hemodynamic responses to speech stimuli. Both genetic and environmental variance differentially appeared for each type of hemoglobin within each bilateral temporal area. Environmental variance in the hemodynamic response to speech was larger than genetic variance, suggesting that environmental factors may play an important role in infants' neural processing of speech stimuli. Moderate and low shared environmental influences on the concentration of oxy-Hb were observed within right and left temporal areas, respectively. This suggests that variations in infants' hemodynamic responses to speech stimuli may be partly dependent on characteristics that are present in the twins and twin siblings environment. A shared environmental influence on language ability has been reported in both infants and children (e.g., Alarcón et al., 1998; Van Hulle et al., 2004). The shared environmental influence on hemodynamic responses to speech stimuli may be consistent with that of language abilities in children.

There are some potential examples of shared environmental factors affecting infants' hemodynamic responses to speech stimuli. One possibility is that the shared environmental factors have been associated with an influence within families. Here, twin infants are reared together in their own family home in which intonation and stress of the Japanese language is homogeneous while matching local dialects. Twin and twin siblings' neural processing of speech stimuli may be sensitive to the same characteristics. Another possibility is that a family-level environmental factor, such as socioeconomic status, which has been previously reported to affect a child's language ability and cognitive function (e.g., Rowe \& Goldn-Meadow, 2009), affects hemodynamic responses to speech stimuli. These fam- ily-level environmental factors may play important roles in an infant's neural processing of speech information.

Unique environmental factors had a much larger influence on hemodynamic responses to speech than did shared environmental factors, suggesting that twin and twin siblings differentially, and individually, develop neural processing within the same environment; here, they share the same family, socioeconomic status, and exposure to acoustic characteristics. Within the same environments, infants' neural processing of speech stimuli may differentially activate. Concrete factors associated with unique environmental variance within infants' neural processing for speech stimuli should be examined in future studies.

In contrast to environmental factors, no substantial additive genetic variance in oxy-Hb within bilateral temporal areas was detected, suggesting that genes may not contribute to infants' acquisition of language ability to the same extent as environmental factors. However, additive genetic factors, which accounted for half of the total variance, were observed within the left temporal area. According to Kato (2004), changes in the concentration of deoxy- $\mathrm{Hb}$ may indicate the neural activation required more deoxy-Hb than oxy-Hb. As such, genetic contributions to infants' neural activity in response to speech stimuli may exist. Furthermore, additive genetic influences on left deoxy-Hb concentration also suggests infants' left cerebral dominance for processing speech despite no differences in the mean hemodynamic response.

Findings of genetic and environmental influences provide new implications for understanding infants' hemodynamic response to speech stimuli. In general, deoxy-Hb concentration is nearly associated with oxy- $\mathrm{Hb}$; thus, oxy- $\mathrm{Hb}$ concentration is considered a useful index of neural activity as opposed to deoxy-Hb concentration. In this study, additive genetic and shared environmental influences appeared within infant deoxy- and oxy-Hb concentrations, respectively. The different variance components among these hemoglobin types suggest that infants' oxy- and deoxy-Hb concentrations indicate different characteristics of neural activity in response to speech stimuli.

Although the grand mean waveforms of hemodynamic responses to speech appeared to deflect within bilateral temporal areas, significant changes in the variation of oxy- and deoxy- $\mathrm{Hb}$ concentration were not observed. In addition, no cerebral dominance or developmental changes in hemodynamic responses for speech stimuli appeared. These results suggest that large individual differences in hemodynamic responses to speech exist during the first two years of life. However, large individual differences are sometimes considered to be meaningless com- 
ponents (e.g., measurement error). Thus, the lack of significant differences in hemodynamic responses to speech appears to provide no clear implications. However, in this study, the individual differences in hemodynamic responses provide valid variance as inferred from the degree of genetic and environmental influences. As mentioned in the introduction section, twin designs decompose variance into additive genetic, shared environmental, and unique environmental variance. Measurement error is only involved in unique environmental variance. In this study, the individual differences in hemodynamic responses to speech have significant variance within the additive genetic and shared environmental factors. Therefore, the large individual differences in hemodynamic responses should be meaningful components for right oxy-Hb and left deoxy-Hb.

This study highlights the genetic and environmental etiology of hemodynamic responses to speech stimuli. The present findings revealed that additive genetic factors, as well as shared and unique environmental factors are significant contributors to each type of hemoglobin within each bilateral temporal area. These results provide new knowledge regarding infants' hemodynamic responses to speech stimuli. Further investigation into the role of genetic and environmental variation within the infant neural system, using a larger sample size, may provide more important information for understanding the mechanisms underlying infant neural maturation.

\section{Acknowledgements}

We are grateful to the families who participated in the study. Data collection for this study was supported by a grant from the Research Institute of Science and Technology for Society within the Japan Science and Technology Agency. This manuscript was supported by Grant-in-Aid for Young Scientists (B) (\#25730101) by the Japanese Ministry of Education, Culture, Sports, Science and Technology.

\section{REFERENCES}

Alarcón, M., Plomin, R., Fulker, D. W., Corley, R., \& DeFries, J. C. (1998). Multivariate path analysis of specific cognitive abilities data at 12 years of age in the colorado adoption project. Behavior Genetics, 28, 255-264. doi:10.1023/A:1021667213066

Ando, J., Nonaka, K., Ozaki, K., Sato, N., Fujisawa, K. K., Suzuki, K., \& Ooki, S. (2006). The Tokyo twin cohort project: Overview and initial findings. Twin Research and Human Genetics, 9, 817-826. doi:10.1375/twin.9.6.817

Boker, S., Neale, M., Maes, H., Wilde, M., Spiegel, M., Brick, T., \& Fox, J. (2011). OpenMx: An open source extended structural equation modeling framework. Psychometrika, 76, 306-317. doi:10.1007/s11336-010-9200-6

Bortfeld, H., Fava, E., \& Boss, D. A. (2009). Identifying cortical lateralization of speech processing in infants using near-infrared spectroscopy. Developmental Neuropsychology, 34, 52-65. doi:10.1080/87565640802564481

Cheour, M., Ceponiene, R., Lehtokoski, A., Luuk, A., Allik, J., Alho, K., \& Näätänen, R. (1998). Development of language-specific phoneme representations in the infant brain. Nature Neuroscience, 1, 351-353. doi:10.1038/1561

Chiron, C., Jambaque, I., Nabbout, R., Lounes, R., Syrota, A., \& Dulac, O. (1997). The right brain hemisphere is dominant in human infants. Brain, 120, 1057-1065. doi:10.1093/brain/120.6.1057

Conboy, B. T., \& Kuhl, P. K. (2011). Impact of second-language experience in infancy: Brain measures of first- and second-language speech perception. Developmental Science, 14, 242-248.
Dehaene-Lambertz, G., Hertz-Pannier, L., Dubois, J., Mériaux, S., Roche, A., Sigman, M., \& Dehaene, S. (2006). Functional organization of perisylvian activation during presentation of sentences in preverbal infants. Proceedings of the National Academy of Sciences of the United States of America, 14240-14245. doi:10.1073/pnas.0606302103

Gilmore, J. H., Schmitt, J. E., Knickmeyer, R. C., Smith, J. K., Lin, W., Styner, M., \& Neale, M. C. (2010). Genetic and environmental contributions to neonatal brain structure: A twin study. Human Brain Mapping, 8, 1174-1182.

Homae, F., Watanabe, H., Nakano, T., \& Taga, G. (2007). Prosodic processing in the developing brain. Neuroscience Research, 59, 29-39. doi:10.1016/j.neures.2007.05.005

Jansen, A., Lohmann, H., Scharfe, S., Sehlmeyer, C., Deppe, M., \& Knecht, S. (2007). The association between scalp hair-whorl direction, handedness and hemipheric language dominance: Is there a common genetic basis of lateralization? NeuroImage, 35, 853-861. doi:10.1016/j.neuroimage.2006.12.025

Kato, T. (2004). Principle and technique of NIRS-Imaging for human brain FORCE: Fast-oxygen response in capillary event. International Congress Series, 1270, 85-90. doi:10.1016/j.ics.2004.05.052

Knecht, S., Dräger, B., Bobe, L., Lohmann, H., Flöel, A., Ringelstein, E.-B., \& Henningsen, H. (2000). Handedness and hemispheric language dominance in healthy humans. Brain, 123, 2512-2518. doi:10.1093/brain/123.12.2512

Kotilahti, K., Nissilä, I., Näsi, T., Lipiäinen, L., Noponen, T., Meriläinen, P., \& Fellman, V. (2010). Hemodynamic responses to speech and music in newborn infants. Human Brain Mapping, 31, 595-603.

Lenroot, R. K., Schmitt, J. E., Ordaz, S. J., Wallace, G. L., Neale, M., Lerch, J. P., \& Giedd, J. N. (2009). Differences in genetic and environmental influence on the human cerebral cortex associated with development during childhood and adolescence. Human Brain Mapping, 30, 164-174. doi:10.1002/hbm.20494

Maki, A., Yamashita, Y., Ito, Y., Watanabe, E., Mayanagi, Y., \& Koizumi, H. (1995). Spatial and temporal analysis of human motor activity using noninvasive NIR topography. Medical Physics, 22, 1997 2005. doi:10.1118/1.597496

Minagawa-Kawai, Y., Cristià, A., \& Dupoux, E. (2011). Cerebral lateralization and early speech acquisition: A developmental scenario. Developmental Cognitive Neuroscience, 1, 217-232. doi:10.1016/j.den.2011.03.005

Neale, M. C., \& Maes, H. H. M. (2004). Methodology for genetic studies of twins and families. Dordrecht: Kluwer Academic Publisher.

Novak, G. P., Kurtzberg, D., Kreuzer, J. A., \& Vaughan Jr., H. G. (1989). Cortical responses to speech sounds and their formants in normal infants: Maturational sequence and spatiotemporal analysis. Electroencephalography and Clinical Neurophysiology, 73, 295-305. doi:10.1016/0013-4694(89)90108-9

Ooki, S., \& Asaka, A. (2004). Zygosity diagnosis in young twins by questionnaire for twins' mothers and twins' self-reports. Twin Research and Human Genetics, 7, 5-12.

Oostenveld, R., \& Praamstra, P. (2001). The five percent electrode system for high-resolution EEG and ERP measurements. Clinical Neurophysiology, 112, 713-719. doi:10.1016/S1388-2457(00)00527-7

Rowe, M. L., \& Goldn-Meadow, S. (2009). Differences in early gesture explain SES disparities in child vocabulary size at school entry. Science, 323, 951-953. doi:10.1126/science. 1167025

Sato, T., Ito, M., Suto, T., Kameyama, M., Suda, M., Yamagishi, Y., \& Mikuni, M. (2007). Time courses of brain activation and their implications for function: A multichannel near-infrared spectroscopy study during finger tapping. Neuroscience Research, 58, 297-304. doi:10.1016/j.neures.2007.03.014

Van Hulle, C. A., Goldsmith, H. H., \& Lemery, K. S. (2004). Genetic, environmental, and gender effects on individual differences in toddler expressive language. Journal of Speech, Language, and Hearing Research, 47, 904-912. doi:10.1044/1092-4388(2004/067) 